\title{
102. Studies on Correlation in Reflective Movements between the Right and the Left Eye with the Use of Newly Devised Recording Method of Nystagmography
}

\author{
Manabi HinokI, and Tsukasa Kurosawa \\ Department of Otolaryngology, Iwate Medical University School of Medicine
}

Up to now eye movements, particularly eye nystagmus have been, as a rule, recorded both simultaneously, but not separately. The authors have had often wondered whether or not in all cases of vertigo, especially in vertigo of central origin, one eye can always move precisely with the other. From this consideration, the authors devised the following method of nystagmography to establish an analytic method of characteristics of vertigo. The recording method is as follows:

To record nystagmus two electrodes are placed at the outer and the inner angle of the right lid and other two electrodes are placed in the same manner as above at the left lid. In addition to the four electrodes mentioned above one electrode is placed on the right and the left forehead as an earth-electrode respectively. After thus prepared, an optic cylinder in front of a subject is rotated for twenty seconds at the rate of one rotation per ten seconds to the right and to the left. During the time of visual stimulation, nystagmus of the right and the left eye are recorded simultaneously, but separately. The results obtained were as follows:

1) In normal adults with healthy ears, there could be no demonstrable differences in frequency, eye speed and direction between the right and the left eye nystagmus.

2) In sufferers from vertigo due to peripheral labyrinthine disorders, the similar results as seen in normal adults were obtained.

3) In patients with central origin vertigo e.g., in patients with head injury, brain tumors and intracranial vascular disorders, there could be often observed notable differences in frequency or in eye speed (in some cases in direction) between the right and the left eye nystagmus.

These findings, the authors consider, indicate that the test reported here can be used as a clinical test to analyze characteristics of vertigo. 